\title{
Comparison of the amplitude of accommodation determined subjectively and objectively in South African university students
}

\begin{tabular}{|c|c|}
\hline \multicolumn{2}{|c|}{$\begin{array}{l}\text { Authors: } \\
\text { Solani D. Mathebula }{ }^{1} \\
\text { Mologadi D. Ntsoane }^{1} \\
\text { Nkalebetja T. Makgaba }^{1} \\
\text { Khensani L. Landela }^{1}\end{array}$} \\
\hline \multicolumn{2}{|c|}{$\begin{array}{l}\text { Affiliations: } \\
{ }^{1} \text { Department of Optometry, } \\
\text { University of Limpopo, } \\
\text { South Africa }\end{array}$} \\
\hline \multicolumn{2}{|c|}{$\begin{array}{l}\text { Corresponding author: } \\
\text { Solani Mathebula, } \\
\text { Solani.Mathebula@ul.ac.za }\end{array}$} \\
\hline \multicolumn{2}{|c|}{$\begin{array}{l}\text { Dates: } \\
\text { Received: } 22 \text { Nov. } 2017 \\
\text { Accepted: } 22 \text { May } 2018 \\
\text { Published: } 30 \text { July } 2018\end{array}$} \\
\hline \multicolumn{2}{|c|}{$\begin{array}{l}\text { How to cite this article: } \\
\text { Mathebula SD, Ntsoane MD, } \\
\text { Makgaba NT, Landela KL. } \\
\text { Comparison of the amplitude } \\
\text { of accommodation } \\
\text { determined subjectively and } \\
\text { objectively in South African } \\
\text { university students. Afr Vision } \\
\text { Eye Health. 2018;77(1), a437. } \\
\text { https://doi.org/10.4102/aveh } \\
\text { v77i1.437 }\end{array}$} \\
\hline \multicolumn{2}{|c|}{$\begin{array}{l}\text { Copyright: } \\
\text { (c) 2018. The Author(s). } \\
\text { Licensee: AOSIS. This work } \\
\text { is licensed under the } \\
\text { Creative Commons } \\
\text { Attribution License. }\end{array}$} \\
\hline \multicolumn{2}{|l|}{ Read online: } \\
\hline 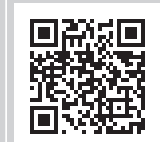 & $\begin{array}{l}\text { Scan this QR } \\
\text { code with your } \\
\text { smart phone or } \\
\text { mobile device } \\
\text { to read online. }\end{array}$ \\
\hline
\end{tabular}

Background: Historically, two clinical methods have been used for measuring the amplitude of accommodation, which are the push-up and minus lens methods. However, it has been documented that the push-up method overestimates amplitude of accommodation, while the minus lens method underestimates it.

Aim: The purpose of this study was to compare subjective and objective procedures for determining the monocular amplitude of accommodation in young optometry students.

Setting: The study was conducted in the optometry clinic at the university.

Methods: Amplitude of accommodation was measured on 45 optometry students (17 males and 28 females, whose ages ranged from 21 to 27 years) using the push-up, push-down, minus lens, modified dynamic retinoscopy and Pascal dynamic retinoscopy methods. Data were collected by three different examiners in this study. One examiner measured all the subjective tests, while another examiner measured the modified dynamic retinoscopy. The third examiner measured the Pascal heterodynamic retinoscopy.

Results: The highest amplitude of accommodation was obtained using the push-up method $(10.23 \pm 1.67 \mathrm{D})$, while the minus lens method gave the lowest subjective finding $(8.43 \pm 1.68 \mathrm{D})$. However, the subjective methods generally produced comparable results. Both retinoscopic methods showed the lowest mean amplitude of accommodation of approximately $6.50 \pm 1.40 \mathrm{D}$. However, there was a high correlation between the various methods.

Conclusion: The push-up and push-down methods overestimate the true amplitude of accommodation because of the relative magnification, while the minus lens method creates an abnormal viewing environment in which the target is stationary but the stimulus becomes increasingly minified. Subjective amplitude of accommodation is an inadequate measure to assess any true accommodation because it fails to differentiate between passive depth of focus and an active accommodative power change in the eye. Therefore, subjective measurement of the amplitude of accommodation may suggest that accommodation is present when it is not. Further research is needed to further validate dynamic retinoscopy as the optimal or best possible routine clinical method to assess the true amplitude of accommodation.

\section{Introduction}

The eye is one of the human body organs which may not effectively carry out its main function of providing clear and comfortable vision even though it appears healthy. 1,2,3 The healthy eye alone does not always guarantee provision of clear and comfortable vision for an individual for a given fixation distance. Accommodation in the eye is one of the systems that play a significant role in the formation of a clear retinal image. ${ }^{1}$ The accommodative system of the human eye is one of the several highly complicated functions necessary to execute and carry out very fine and detailed near work.

Accommodation can be defined as an increase in the dioptric or refractive power of the eye to focus clearly on objects at various distances. ${ }^{1,2,3,4,5,6,7}$ An increase in the optical system of the eye occurs because of an increase in the anterior and posterior surface curvatures of the crystalline lens resulting from contraction of the ciliary muscle. . $^{8,910,11,12,13}$ The radius of curvature of the anterior surface of the crystalline lens reduces by $0.33 \mathrm{~mm}$ per dioptre of accommodation, while the posterior surface reduces by $0.15 \mathrm{~mm}$ per dioptre of accommodation. ${ }^{4}$ 
The greatest increase in refractive change an eye can undergo or the maximum amount of accommodation that can be exerted is called the amplitude of accommodation or accommodative amplitude. ${ }^{1,2,3,4,5,6,7}$ Amplitude of accommodation (AA) changes as a function of age from at least the early teenage years, , $^{14,15,16,17,18,19,20,21,22,23,24}$ with presbyopic symptoms starting to occur at about 40 to 45 years of age, when the accommodative reserve becomes insufficient to maintain focus on near objects. The pre-presbyopic symptoms manifest early in hyperopes and in emmetropes at about 40 years of age. ${ }^{21}$ The loss of AA progresses until about $55-60$ years when accommodation is completely lost or a presbyopic person has essentially zero AA. ${ }^{24}$ However, subjective measurement methods imply that some AA (approximately $1 \mathrm{D}$ ) persists beyond 60 years of age, whereas objective findings indicate that the AA reaches zero around 55 years of age..$^{21,22,24}$

Amplitude of accommodation is measured clinically using various subjective methods (push-up, push-down and minus lens). ${ }^{21,22,23,24}$ Although these measurements provide important information about the AA, they do not accurately measure the accommodative optical change that occurs in the eye. Recent studies have utilised objective methods to quantify the magnitude of refractive change of the eye to more accurately and precisely depict accommodative ability. $25,26,27,28,29$ These studies demonstrate that the subjective methods overestimate the true AA of the eye because of depth of focus, target size, illumination, end-point criteria, proximal cues, pupil size and subject variability. $30,31,32,33,34$

Objective tests of the AA can possibly differentiate true AA from pseudo accommodation or other possible confounding factors. In view of the paucity of studies, the purpose of this study was to compare subjective and objective methods of stimulating and measuring AA in young pre-presbyopic (21-27 years old) optometry students to understand the benefit and drawbacks of each method. Given the ease of performing the subjective measurements, a useful conversion equation can be derived to convert the clinically utilised subjective measurement to accurately approximate AA values determined objectively.

\section{Methods}

This study was carried out in the optometry clinic at the university between March and July 2017. Subjects included 17 males and 28 females ranging in age from 21 to 27 years. Measurements of the AA were obtained from 45 healthy final-year optometry students that met the inclusion criteria. Informed consent was obtained from each subject after a thorough explanation of the purpose, objectives, procedures and the possible results. Subjects were given an opportunity to ask about the research study. The study was conducted according to the tenets of the Declaration of Helsinki. The inclusion criteria included subjects with visual acuity of $6 / 6$ or better in each eye at $6 \mathrm{~m}$ and $0.4 \mathrm{~m}(40 \mathrm{~cm})$ with no amblyopia, strabismus, history of corneal trauma or ocular pathology and not taking any medications which are known for interfering with accommodation.
Before measuring and recording the AA measurements, the refractive error of each subject was determined using static retinoscopy and subsequently refined with the subjective refraction (including the Jackson cross cylinder method) and balanced using the prism dissociation test. Subjects included 31 emmetropic, 3 myopic, 7 hyperopic and 4 astigmatic participants. The measured refractive correction for each participant was worn for all AA measurements because of the fact that a myope would give a false high reading and a hyperopia a low one. ${ }^{1,2,3}$ Monocular AA was then measured using the five different methods: three subjective (push-up, push-down and minus lens-to-blur) and two objective (modified dynamic retinoscopy and Pascal heterodynamic retinoscopy) methods. AA data were obtained by three experienced optometrists (LM, NM and MN). Results were recorded by an assistant research student. Each test was performed monocularly but only the results of the right eyes are presented here.

The procedures were performed in a triple masked fashion by experienced clinicians with more than 15 years of experience to prevent inter-examiner variability. One examiner performed the subjective testing of AA, whereas the other two examiners determined the AA objectively in all subjects. $\mathrm{MN}$ always determined the AA using the modified dynamic retinoscopy method and NM the Pascal dynamic retinoscopy method. Neither examiner knew the other examiner's results. The endpoint criteria were defined as the neutral reflex when no movement could be seen. To assess the repeatability, a pilot study was conducted on five subjects drawn from the thirdyear optometry students. Measurements were performed using all five procedures by a single examiner (SD). The five methods are described individually below.

\section{Push-up method}

This is the most common and simplest method to measure the AA. It is also called the Donder's method because it was first described by Donders in $1864 .{ }^{14,31}$ In this method, the target (line of letters) through the appropriate distance correction is moved towards the patient until blur of the target is reported. ${ }^{24,32,35,36,37,38,39,40,41}$ In this study, the refractive correction was placed in a trial frame. With the left or right eye occluded, the subject's attention was directed to a 20/20 line of letters on a handheld reduced Snellen chart at a distance of approximately $40 \mathrm{~cm}$. Subjects or participants were instructed to keep the letters as clear as possible and to report when letters became blurred. The target was then gradually moved at a rate of approximately $5 \mathrm{~cm} / \mathrm{s}$ towards the subject's fixing eye until sustained blur of the letters was reported. Subjects were repeatedly asked if the target was still clear as it was moved towards their eyes and to report immediately as soon as it became a little bit blurry or fuzzy. The end-point was the first, slight, sustained blur, which could not be cleared after 2 or $3 \mathrm{~s}$ of viewing. ${ }^{1}$ When the first slight sustained blur was achieved, the target is at the eye's near point. The distance from the target (where blur was sustained) to the spectacle plane was measured with a millimetre ruler and converted to dioptres, and recorded as 
the subjective AA. The test was performed twice per eye and the average result was recorded as the amount of the AA. The level of illumination remained relatively constant by moving the overhead lamp as the target was moved. (The overhead lamp was being moved by the assistant research student as the examiner was performing the procedure).

\section{Push-down method}

The push-down or pull-away method is a variation or alteration of the push-up method in which the target was placed very close to the subject and then slowly pushed away until the target could be identified. ${ }^{35,36,37,38,39,40,41,42}$ With the distance refractive correction placed in a trial frame and the left or right eye occluded, the accommodative target (20/20 line of letters) was initially positioned close to the trial frame and subjects were asked to push the handheld reduced Snellen chart away at a rate of approximately $5 \mathrm{~cm} / \mathrm{s}$ until a 20/20 line of letters could just be seen clearly and sharply or become readable. Again, the distance from the target to the spectacle plane was measured and converted to dioptres. This procedure was performed twice per eye and the average result recorded as the AA. This procedure took approximately $30 \mathrm{~s}$ to complete per subject. The speed of the target was the same as that for the push-up method. The overhead lamp was moved by the assistant research student.

\section{Minus lens-to-blur method}

The distance refractive correction was introduced into the phoropter and the test was performed monocularly. A reduced Snellen chart was placed in front of the phoropter at a fixed viewing distance of $40 \mathrm{~cm}$, corresponding to a stimulus of $2.50 \mathrm{D}$. Subjects were instructed to keep the illuminated letters clear and sharp and to report the first noticeable sustained blur that could not be cleared by further conscious effort. Minus lenses in $0.25 \mathrm{D}$ steps were introduced over the distance correction. When the letters became and remained blurred, the AA was recorded as $2.50 \mathrm{D}$ (the dioptric equivalent of the working distance) plus the amount of minus lens power added, ignoring the minus sign. It took about $1 \mathrm{~min}$ to complete this procedure per participant.

The minus lens method is routinely performed with the target at $40 \mathrm{~cm}$; however, some authors prefer $33 \mathrm{~cm} \cdot{ }^{40,41,42,43,44,45}$ Placing the target at $33 \mathrm{~cm}$ rather than $40 \mathrm{~cm}$ is believed to make the target appear smaller and may make the patient more sensitive to identifying the first noticeable blur, and this may reduce the possibility of getting underestimated AA. Scheiman and Wick ${ }^{46}$ believes that placing the target at $33 \mathrm{~cm}$ is done to compensate for the effect of minification but only 2.50 D not 3.00 D should be added to the obtained AA result. However, in this study a $40 \mathrm{~cm}$ distance was used and $2.50 \mathrm{D}$ was added not $3 \mathrm{D}$.

The push-up and the push-down methods can all be measured under monocular and binocular conditions but the minus lens method should only be performed under monocular conditions because it can result in an excess of accommodative convergence which could disrupt the binocularity. ${ }^{46}$

\section{Modified dynamic retinoscopy}

The test was performed monocularly in a dimly illuminated room and with the subject wearing the distance refractive correction. Each subject looked at the front-illuminated near point card with paragraph text as an accommodative stimulus attached to the front of the streak retinoscopy at $40 \mathrm{~cm}$. Each subject was instructed to read the letters and keep them clear. Although the subject read the letters aloud, the examiner $(\mathrm{MN})$ used the vertical streak to perform the test. When a with movement (lag of accommodation) was observed, the examiner moved the retinoscope inward until neutral reflex was first observed..$^{47,48,49}$ Once the neutrality was achieved, the distance between the spectacle plane and retinoscope was measured with a tape measure. The modified dynamic retinoscopy was taken as the reciprocal or inverse of the distance in metres. The test was performed similarly for the left eye.

The principle of this method is that a neutral reflex will be observed when the point conjugate with the retina coincide with the plane of the retinoscope sighthole. . $25,47,48,49$ When a 'with' movement is seen, the eye is under-accommodating for the distance of the retinoscope. The examiner then adjusted the working distance by moving forward and backward until neutral motion was observed.

\section{Pascal heterodynamic retinoscopy}

The distance refractive correction was again placed on the trial frame with the left eye occluded. The fixation target was placed close to the trial frame where letters were blurry and then subjects were asked to push the handheld reduced Snellen chart away until the letters were just legible or readable. Subjects were instructed to keep letters sharp and clear. ${ }^{50}$ With the target at this subjective position, the examiner (NM) positioned the retinoscope at a working distance approximately twice the distance between the fixation chart and the subject. The retinoscopy reflex was observed, and if an 'against' movement was seen, the examiner moved closer to the eye until a neutral reflex was found. Once the neutral reflex was observed, the distance between the spectacle plane and the retinoscope was measured with a measuring tape. The objective AA was then taken as the reciprocal of that distance in metres.

An 'against' movement implies that the subject's retinal conjugate point is somewhere behind the target, but in front of the retinoscope and by moving forward, one is finding that the neutral and the conjugate point of near point of accommodation is there. ${ }^{23}$ Each measurement took approximately $90 \mathrm{~s}$ to complete per subject. Again, no working distance lens power was added or subtracted from the distance correction. 


\section{Data analysis}

Descriptive statistical analysis of the data was performed using SPSS version 23. The $t$-test and correlation analysis were used to compare the mean findings from the push-up, push-down, minus lens, modified retinoscopy and Pascal heterodynamic retinoscopy. The normality of the data was checked by using the Shapiro-Wilk test. The KolmogorovSmirnov test should not be seriously considered for testing normality because of its low power. ${ }^{51,52}$ Regression analysis was performed. The differences between the findings for the five methods were compared using Bland-Altman plots. A $(p=) 5 \%$ level of statistical significance was used throughout.

\section{Results}

A total of 45 university students aged between 21 and 27 years with a mean age of 22 years and standard deviation of 6 years were included in the study. Based on subjective refraction, subjects were classified according to the spherical equivalent as 31 emmetropic $(-0.25$ to $+0.50 \mathrm{D}), 7$ hyperopic $(\geq+0.50 \mathrm{D}), 3$ myopic $(\leq-0.25 \mathrm{D})$ and 4 astigmatic $(\geq 0.50 \mathrm{D})$. From the 45 students, 28 (62.2\%) were females and 17 (37.8\%) males. The independent sample $t$-test did not show any significant difference between the mean ages of females and males $(p>0.05)$.

The descriptive analysis for the push-up, push-down, minus lens, modified dynamic retinoscopy and Pascal heterodynamic retinoscopy procedures is shown in Table 1. The Shapiro-Wilk test showed that the measurements of the AA were distributed normally with a $p>0.05$. For the right eyes only, the average AA ranged from $6.58 \mathrm{D}$ to $10.22 \mathrm{D}$; the push-up procedure had the highest average, while the modified dynamic retinoscopy had the smallest means.

Visual inspection of the measurement distributions may be used for assessing normality. The box plot (or box and whisker plot) in Figure 1 indicates the distributions of the AA measurements. Most of the push-down, minus lens, modified dynamic retinoscopy and Pascal dynamic retinoscopy measurements were below $10 \mathrm{D}$, whereas most of the push-up measurements were more than $10 \mathrm{D}$. The box plot indicated that the distributions are roughly symmetrical, and we, therefore, expect the population distribution to be approximately normal. A symmetric box plot with the median line at approximately the centre of the box and with symmetric whiskers that are slightly larger than the subsections of the centre box suggests that the data may have come from a normal distribution. The horizontal bold line in the middle of the plot represents the median or the 50th percentile of each distribution (see Table 1). The box itself represents the middlemost $50 \%$ of the distribution. The box has 'whiskers' (i.e. the vertical lines), one below the first and one above the third quartiles. The whiskers indicate the smallest and largest measurement in each distribution. The push-up and minus lens methods had the longest whiskers above the third quartile. All the methods had slight negative kurtosis but only the push-up and minus lens methods had slight positive skewness (see Table 1).

Table 2 lists the mean differences, standard deviations and $95 \%$ confidence intervals (CIs) for paired comparisons of the

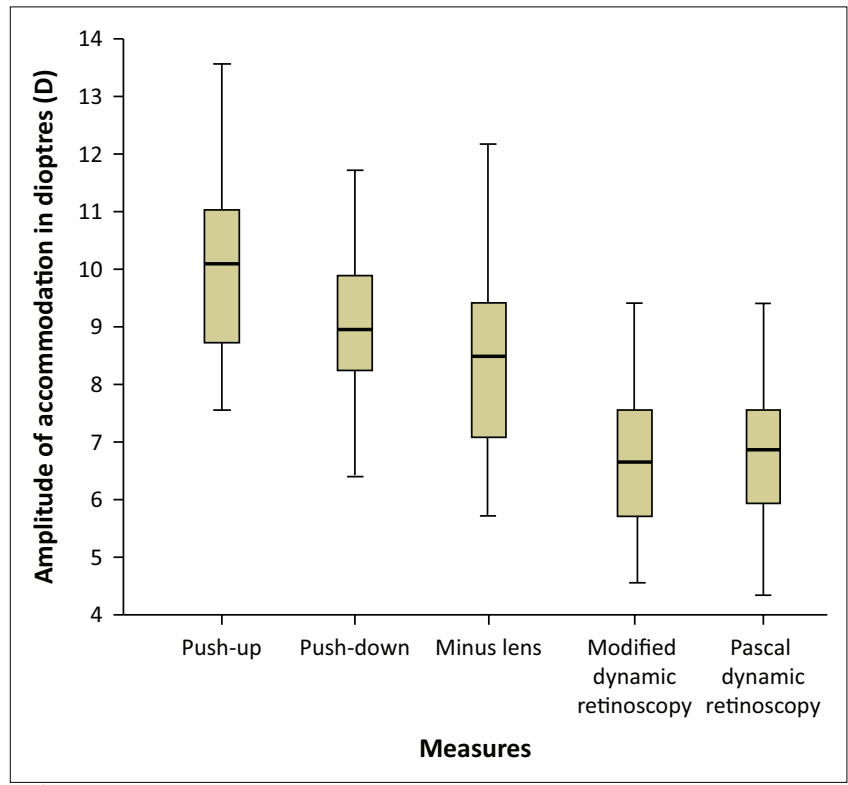

D, dioptre.

FIGURE 1: Box plots of the different measures of the amplitude of accommodation. The box plots display the distributions of the amplitude of accommodation measurements based on the minima, first quartile, second quartile, third quartile and the maxima per sample. Horizontal bold lines inside the boxes show the medians, and whiskers above and below the boxes show the location of the minima and maxima. The interquartile range (IQR) spans the first and third quartiles.

TABLE 1: Descriptive statistics of the measurements of the amplitude of accommodation using the push-up, push-down, minus lens, modified dynamic retinoscopy and Pascal dynamic retinoscopy methods.

\begin{tabular}{|c|c|c|c|c|c|}
\hline Statistics & Push-up & Push-down & Minus lens & Modified DR & Pascal DR \\
\hline Means & 10.22 & 9.08 & 8.43 & 6.58 & 6.77 \\
\hline Standard deviations & 1.67 & 1.44 & 1.68 & 1.34 & 1.42 \\
\hline $95 \%$ Confidence intervals & $9.72-10.72$ & $8.65-9.51$ & $7.93-8.94$ & $6.20-7.00$ & $6.40-6.80$ \\
\hline Medians & 10.25 & 9.00 & 8.50 & 6.50 & 6.75 \\
\hline Minimums & 7.50 & 6.25 & 5.50 & 4.25 & 4.00 \\
\hline Maximums & 14.00 & 12.00 & 12.50 & 9.50 & 9.50 \\
\hline Skewness & 0.35 & -0.03 & 0.20 & 0.04 & 0.04 \\
\hline Kurtosis & -0.43 & -0.54 & -0.54 & -0.72 & -0.50 \\
\hline First quartiles & 8.75 & 8.13 & 6.88 & 5.50 & 5.75 \\
\hline Second quartiles & 10.25 & 9.00 & 8.50 & 6.50 & 6.75 \\
\hline Third quartiles & 11.25 & 10.00 & 9.63 & 7.23 & 7.50 \\
\hline
\end{tabular}

Note: The units are dioptres (D) throughout.

$\mathrm{DR}$, dynamic retinoscopy. 
five methods. The mean differences gave an idea of how much difference there is between the averages of the different methods. The highest mean difference was between push-up and modified dynamic retinoscopy (3.63 D), push-up and Pascal dynamic retinoscopy (3.45 D), push-down and modified DR (2.49 D), and push-down and Pascal dynamic retinoscopy $(2.31 \mathrm{D})$. The results obtained using the subjective methods were higher than those obtained using the objective methods.

In order to establish whether relationships existed between the five methods, a correlation analysis was carried out (see Table 3). The correlation coefficient $(r)$ provides an indication of the linear association between two variables. Correlation coefficients showed significant correlations between the AA measurements. Correlation quantifies the strength of the linear relationship, while correlation coefficient measures the strength of the linear relationship. The value of $r$ is between 1 and -1 . Values of $r$ closer to 1 or -1 represent a strong linear relationship, while a value of $r$ closer to 0 means the linear association is very weak. Correlation coefficient can be interpreted only if the $p$-value is significant and conclude that there is no relationship between the variables because the calculated coefficient of variation (which indicates the absence of correlation) is statistically significant.

The regression analysis between the five procedures and their linear regression models is also presented on each graph

TABLE 2: Comparison of the amplitude of accommodation measurements between five different methods.

\begin{tabular}{lccccc}
\hline Paired procedures & $\begin{array}{c}\text { Mean } \\
\text { differences }\end{array}$ & SD & \multicolumn{2}{c}{$\begin{array}{c}95 \% \text { Cls on mean } \\
\text { differences }\end{array}$} & $p$ \\
\cline { 3 - 5 } & & & Lower & Upper & \\
\hline Push-up and push-down & 1.14 & 0.88 & 0.87 & 1.410 & 0.00 \\
Push-up and minus lens & 1.80 & 1.04 & 1.47 & 2.100 & 0.00 \\
Push-up and modified DR & 3.63 & 1.35 & 3.23 & 4.040 & 0.00 \\
Push-up and Pascal DR & 3.45 & 1.26 & 3.07 & 3.830 & 0.00 \\
Push-down and minus lens & 0.64 & 0.56 & 0.48 & 0.810 & 0.00 \\
Push-down and modified DR & 2.49 & 1.14 & 2.15 & 2.840 & 0.00 \\
Push-down and Pascal & 2.31 & 1.11 & 2.00 & 2.650 & 0.00 \\
Minus lens and modified DR & 1.85 & 1.19 & 1.49 & 2.210 & 0.00 \\
Minus lens and Pascal DR & 1.67 & 1.23 & 1.30 & 2.040 & 0.00 \\
Modified DR and Pascal DR & -0.18 & 0.81 & -0.43 & 0.006 & 0.13 \\
\hline
\end{tabular}

$\mathrm{SD}$, standard deviation; DR, dynamic retinoscopy; Cls, confidence intervals.

TABLE 3: Direct comparison between the individual procedures for the amplitude of accommodation.

\begin{tabular}{lcc}
\hline Correlation & Correlation coefficient $(\boldsymbol{r})$ & Significance level \\
\hline Push-up and push-down & 0.85 & 0.000 \\
Push-up and minus lens & 0.81 & 0.000 \\
Push-up and modified DR & 0.62 & 0.000 \\
Push-up and Pascal DR & 0.68 & 0.000 \\
Push-down and minus lens & 0.95 & 0.000 \\
Push-down and modified DR & 0.67 & 0.000 \\
Push-down and Pascal DR & 0.70 & 0.000 \\
Minus lens and modified DR & 0.71 & 0.000 \\
Minus lens and Pascal DR & 0.70 & 0.000 \\
Modified DR and Pascal DR & 0.83 & 0.000 \\
\hline
\end{tabular}

Note: For all cases, the significance is less than 0.05 , which indicates that there are linear relationships between paired variables.

DR, dynamic retinoscopy. (see Figures 2-4). Regression is about pattern and possible relationship between two sets of data using scatter plots. In actual fact, linear regression is performed together with correlation analysis. It finds the best line that predicts one variable from the other one and quantifies goodness of fit with the coefficient of determination $\left(r^{2}\right)$. The coefficient of determination explains the proportion of variance that the two variables have in common.

Correlation analysis is not the most appropriate method to evaluate agreement between two tests. Tests that supposedly measure the same quantity would be expected to show an association by correlation, but this correlation does not imply agreement. The test of significance may show that two methods are related; however, this could be misleading. Bland-Altman analysis is a method for comparing different measurement methods of the same or different clinical variables. The method also includes horizontal lines to denote $95 \%$ limits of agreements (LoAs). ${ }^{53,54}$

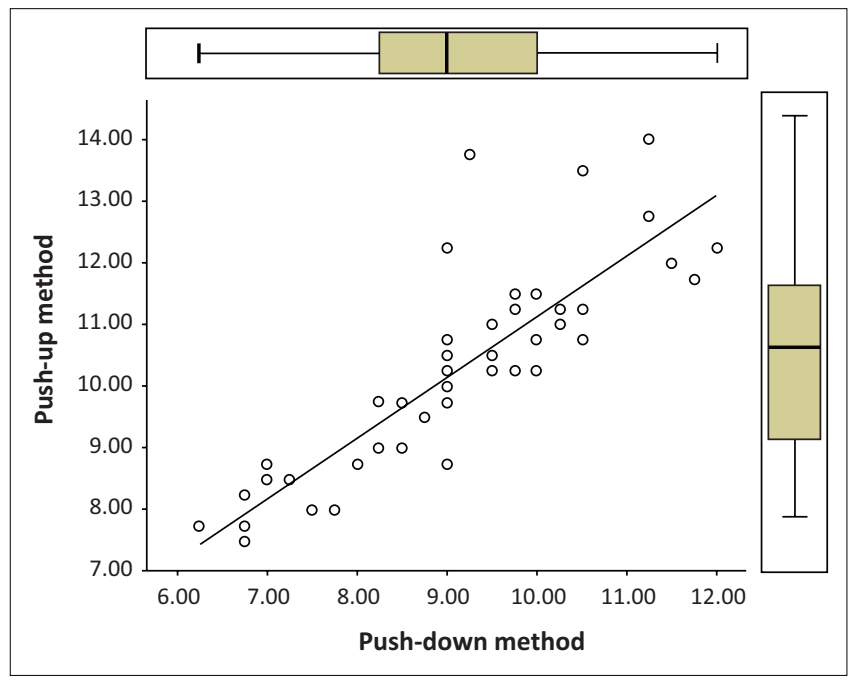

FIGURE 2: Scatter plot of push-down against push-up techniques, with the line of equality indicated. The regression equation is expressed as follows: pushdown $=1.62+0.73$ (push-up). Correlation coefficient between the two methods was $r=0.85(p=0.00)$.

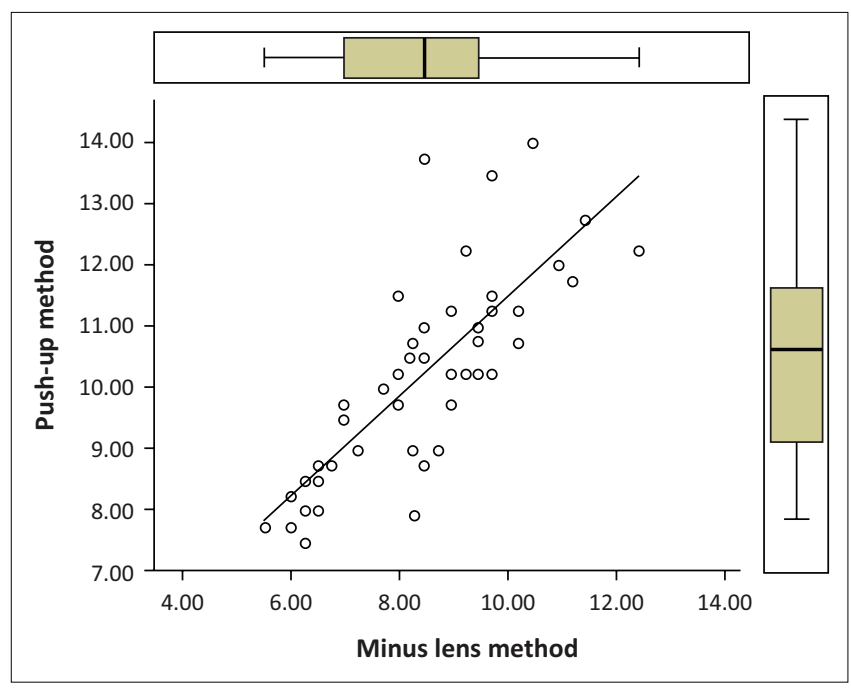

FIGURE 3: Scatter plot comparing the amplitude of accommodation measured subjectively using the push-up and minus lens methods. The correlation coefficient was $r=0.81(p=0.00)$. 


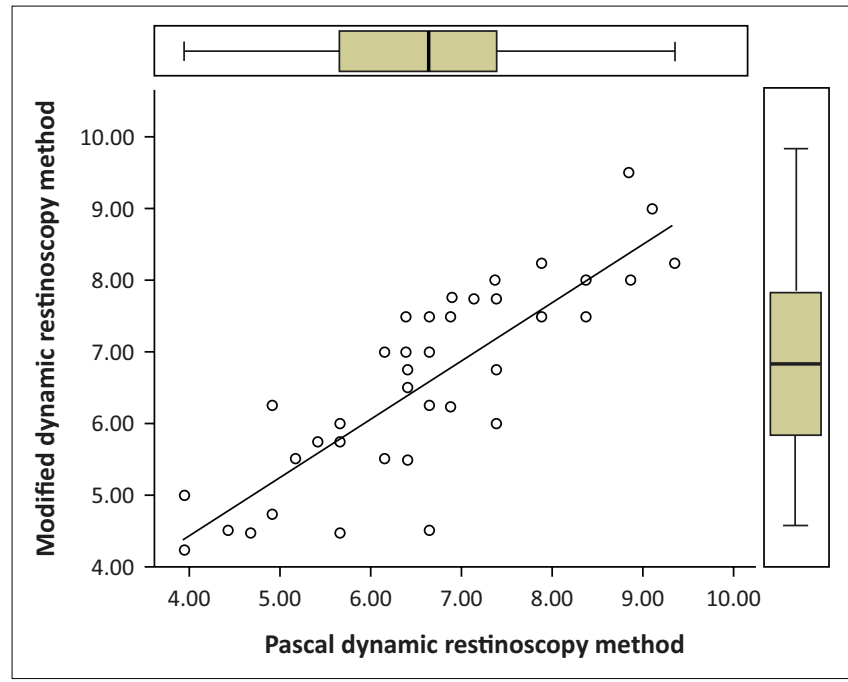

FIGURE 4: Scatter plot of modified dynamic retinoscopy against Pascal dynamic retinoscopy techniques, with the line of equality indicated. Correlation coefficient between the two methods was $r, 0.83(p=0.00)$. The regression equation is expressed as modified DR $=1.199+0.796$ (Pascal DR).

Figures 5 and 6 represent the Bland-Altman plots for two comparisons only. The means for paired amplitudes of accommodation for participants are plotted against their corresponding mean differences. Three horizontal lines are drawn. One is drawn at the mean difference and two at the limits of agreement, which are defined as the mean difference plus and minus 1.96 standard deviation of the differences. About $95 \%$ of the data points will be within the mean difference \pm 1.96 standard deviations.

The 95\% limits of agreements are meant to be estimates of the range in the population of which $95 \%$ of the difference between two measurements lie. As the estimates of the LoAs are based on sample statistics, they might be associated with some uncertainty and thus should be accompanied by an estimate of confidence intervals. To calculate the confidence of intervals for LoAs involves using two-sided tolerance factor for a normal distribution. ${ }^{46,47}$ The confidence interval closer to the mean difference (inner confidence interval) is calculated using coefficient 0.025 , while for outer confidence we used the coefficient 0.975 from the coefficients for $95 \%$ LoAs of the $t$ distribution. Furthermore, the 95\% CIs of the upper and lower limits of agreement were calculated as the limits of agreement \pm 1.96 times the standard error. The standard error of these limits was calculated from the formula:

$$
\sqrt{2.92} \frac{s_{\text {diff }}}{\sqrt{n}}
$$

where $n$ is the sample size, and $s_{\text {diff }}$ is the standard deviation of the differences. As the limits of agreement are only estimates, CIs should be calculated and reported. Confidence intervals describe the range over which a parameter is likely to lie with a given probability of $95 \%$. To obtain the confidence interval, the standard error is calculated using the $t$ distribution table. The limits of agreement for a sample are only an estimate of the LoAs for the population from which the sample is drawn. It is imperative to have an understanding

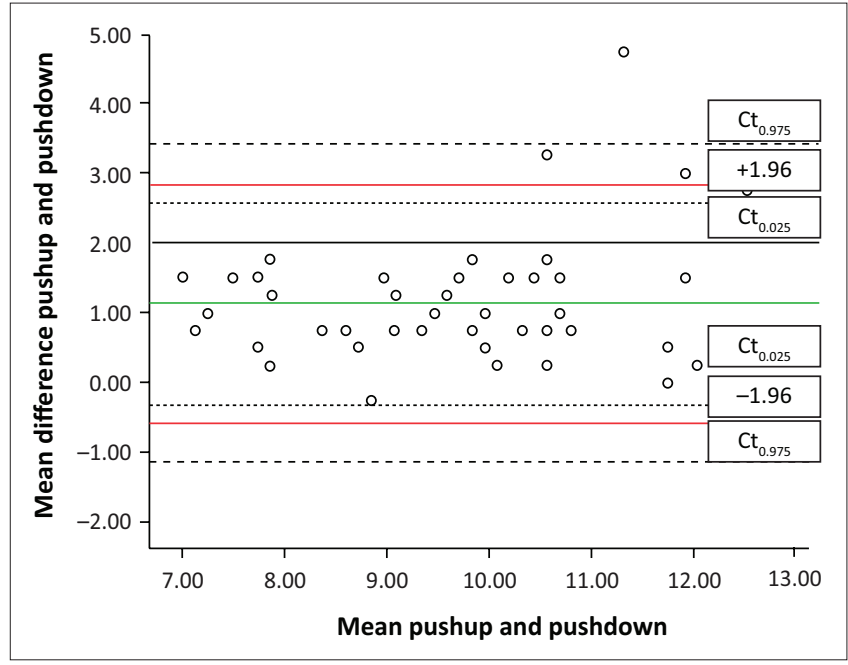

Ct, confidence intervals.

FIGURE 5: A Bland-Altman plot of differences in push-up minus push-down measurements against the means for paired measurements (for each participant concerned) is indicated, together with $95 \%$ confidence intervals about the upper and lower limits of agreement. The limits of agreement are indicated by the red lines, while the dashed and dotted lines are 0.025 (inner) and 0.975 (outer) confidence intervals. There is fairly good agreement between the push-up and push-down measurements. The mean difference is slightly larger than $1 D$ (see Table 2), and the width of the $95 \%$ LoA interval is approximately $3 \mathrm{D}$.

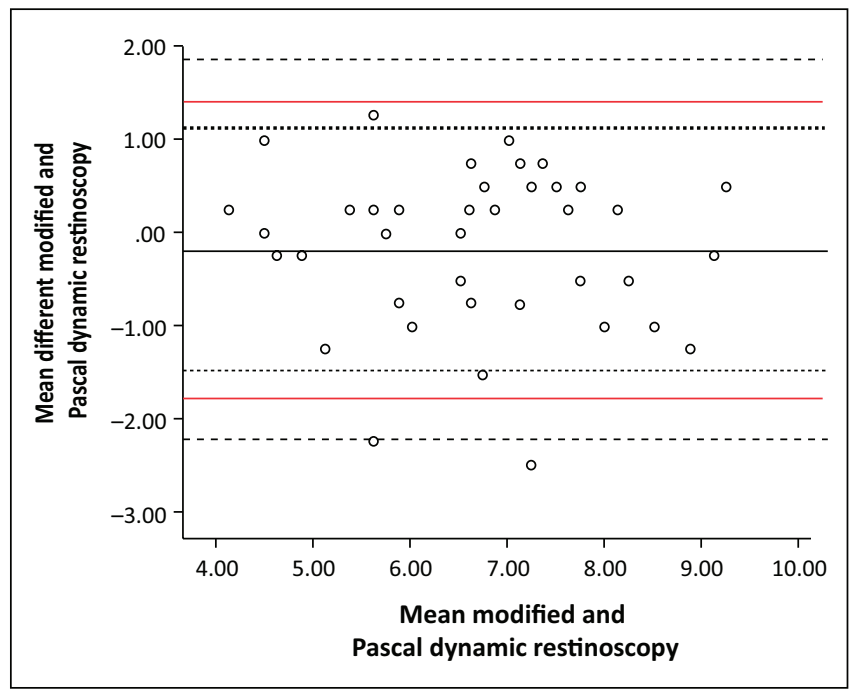

FIGURE 6: A Bland-Altman plot of differences in modified minus Pascal dynamic retinoscopy measurements against the means for paired measurements is indicated, together with confidence intervals on the limits of agreement. There is a good agreement between the measurements from the two methods concerned. The limits of agreement are indicated by the red lines, while the dashed and dotted lines are 0.025 (inner) and 0.975 (outer) confidence intervals.

of how much the LoAs in the population may vary from the sample limits of agreements.

\section{Discussion}

This study involved subjective and objective measurements of AA. The subjective methods showed higher mean amplitudes of accommodation. The push-up method showed the highest mean amplitude of accommodation, while the minus lens method exhibited the lowest mean AA when determined subjectively. The objective measurement of the AA underestimated the subjective AA. The amount of underestimation was about $3.60 \mathrm{D}$ (see Table 1 and Figure 1). 
The most widely used clinical procedures to assess the AA are the subjective push-up and the minus lens methods. Both methods require an individual who is corrected for best distance visual acuity. Although a subjective method provides important information about vision comfort and sustainability during near visual tasks, it does not necessarily accurately measure the accommodative optical change that occurs in the eye because of the eye's depth of field that causes subjective measurements to overestimate the objectively measured AA. ${ }^{24,26}$ The differences between the objective and subjective methods can be explained by the lag of accommodation. Subjective assessment measures the closest distance at which the patient can see clearly. The objective methods evaluate the actual increase in refractive power of the eye. The lag of accommodation increases with the accommodative stimulus. This could probably be because of pupillary miosis which increases the depth of focus. According to Hokoda and Ciuffreda, ${ }^{23}$ the difference between the subjective and objective methods can vary by as much as $1.50 \mathrm{D}-2.0 \mathrm{D}$, which is less than what was obtained in this study. The difference between subjective and objective methods in this study varied between $1.8 \mathrm{D}$ and 3.6 D.

The subjective push-up method may be adequate for routine use to measure AA but it is inadequate for measuring true AA as it overestimates the AA. The higher values seen when measuring AA with the push-up methods in comparison with other methods have been documented. This has been attributed to the depth of focus, target size, illumination, proximal cues, pupil size, end-point criteria and subject variability. ${ }^{26,27,33,37,39}$ When performing the push-up method, there is an increase in the angular size of the retinal image corresponding to the decrease in the target distance and also the proximal stimulation to the accommodation increases and leads to a higher value compared to other methods. This increase in angular subtense may result in a delay in subject's ability to report the end-point which is blur. The end-point of first sustained blur can also be a difficult concept for some patients to appreciate. Illumination can also affect measurements. The target should be illuminated by a 40 -watt incandescent bulb. ${ }^{31}$ Excessive illumination can greatly increase the depth of focus for some patients and result in false high AA measurements. Chen and $\mathrm{O}^{\prime}$ Leary ${ }^{38}$ compared measurements of AA using the push-up and modified pushup methods in a sample of 29 young subjects. Higher values were recorded under monocular and binocular conditions for the push-up method.

The results of this study showed that the minus lens method had the lowest mean AA among the subjective methods. This result is in agreement with the results from previously documented studies. ${ }^{21,24,29,32}$ In the minus lens method, unlike in the push-up method, there is minification of the retinal images because of the optical properties of the higher powered minus spherical lenses while there is no relative distance magnification, and the proximal stimulation of the accommodation remains constant. ${ }^{1}$ This explains why the push-up amplitude result is higher than the minus lens amplitude. Based on factors affecting the subjective AA measurement, the minus lens method may be a better (or appropriate) and accurate method to measure the AA. However, the push-up method is faster and more widely used than the minus lens-to-blur method.

The push-down or pull-away method for AA is one of the newer and less researched methods in the literature. This method requires a target to be pushed away from the patient's spectacle plane until the target can be correctly identified. The results of this study showed a difference of $1 \mathrm{D}$ between push-up and push-down and $0.7 \mathrm{D}$ between push-down and minus lens methods. The differences between the three subjective amplitude were small but statistically significant differences (see Table 2). Results of this study are in agreement with the results of Antona et al..$^{40}$ who showed an average difference of between $0.50 \mathrm{D}$ and $2.50 \mathrm{D}$ between push-up, push-down and minus lens methods. Pollock, ${ }^{37}$ Woehrle et al., ${ }^{36}$ and Chen and $\mathrm{O}^{\prime}$ Leary ${ }^{38}$ compared the push-up and push-down testing methods. They found that there was no statistical significant difference between the push-up and push-down methods. However, Rosenfield and Cohen ${ }^{39}$ found significant differences among the push-up, pushdown and minus lens methods. The possible explanation to this contrasting conclusions could be the end-point required. When performing the push-up, the end-point required is the first slight sustained blur. In the Rosenfield and Cohen's study ${ }^{39}$, subjects were to wait until the target was absolutely clear. In this study and for that by Woehrle et al. ${ }^{36}$, participants had to identify the target at the first possible clarity. However, in Pollock's ${ }^{37}$ study subjects were required to report clarity or complete blur.

Our results showed that the push-down test consistently give lower results of AA when compared to push-up testing. One possible reason for this difference could be the psychophysical testing procedure which operates in opposite directions for the two methods. The push-up method would overestimate the AA, while the push-down method would minimise the amplitude. Perhaps in the push-down testing method, the end-point is more easily understood. However, this has not yet been shown to be true. It is possible that it is easier to recognise the point of identification in the push-down method than the point of first sustained blur. If the pushdown method is to be used more extensively in clinical practice, it is also necessary to conduct a study on a large sample to determine normative data.

Recently, there has been studies comparing subjective (push-up, push-down, minus lens and defocus) and objective (aberrometer or autorefractor) methods of measuring the AA. ${ }^{24}$, $25,26,28,55,56,57,58,59,60,61$ Results of these studies showed that the subjective methods overestimated the AA. The limitations of subjective methods have already been documented in this article.

An objective method determines the end-point of AA by observation and interpretation of the retinoscopic reflex. However, such objective measurements are not yet widely 
used in optometric practice. Objective methods of measuring AA measure the actual increase in refractive power of the eye, while the subjective methods measure the closest distance at which the patient can see clearly. This can exceed the near point of accommodation by approximately half the total depth of field of the eye. ${ }^{45}$ Therefore, the difference between subjective and objective methods can vary by as much as $1.8 \mathrm{D}-3.6 \mathrm{D}$.

The two dynamic retinoscopy findings showed the lowest mean AA of the five methods used in this study. The means, standard deviations and ranges of the AA measured using the modified dynamic retinoscopy and Pascal dynamic retinoscopy did not vary much (see Table 1 and Figure 1). Hokoda and Ciuffreda ${ }^{23}$ compared AA measured with an objective Pascal dynamic retinoscopy method and with subjective minus lens and push-up methods in seven amblyopes. They found that the mean AA obtained using Pascal dynamic retinoscopy was lower than the findings obtained using either the push-up (mean difference $=2.40 \mathrm{D}$ ) or the minus lens (mean difference $=0.77 \mathrm{D}$ ) methods in the control subjects. However, in the amblyopic eyes, the findings were variable. The difference between the mean objective finding and the push-up method was $5 \mathrm{D}$ in the push-up method but the DR findings were $0.46 \mathrm{D}$ higher than the minus lens method.

Rutstein et al. $^{25}$ compared AA determined objectively and subjectively in a sample of 54 subjects aged between 6 and 35 years using the push-up method and modified DR. They found that the modified DR consistently gave higher mean values than the push-up method. The reason of a higher finding for AA measured objectively could be the end-point criterion. Rutstein et al. ${ }^{25}$ defined the end-point when the width of the retinoscopy reflex became narrow, its colour became dimmer and its speed became slower. This end-point criterion could be the cause of the higher values in modified DR. In this study, we used the more commonly adopted neutral reflex for the end-point.

Woodhouse et al. ${ }^{61}$ measured AA in children with Down syndrome using dynamic retinoscopy. Their end-point criterion was the position of the neutralisation, as in the current study. They found no significant difference between the push-up and DR results. Leon et al. ${ }^{44,45}$ examined the reliability of Pascal dynamic retinoscopy and two subjective (modified push-down and minus lens) measurements of AA in a sample of 79 optometry students between 18 and 30 years of age. They found that the Pascal dynamic retinoscopy method showed higher reproducibility when compared with subjective methods. Also, they observed that the dynamic retinoscopy method provides more veridical measurements of the AA as it avoids the overestimation because of the depth of field. Anderson and Stuebing ${ }^{22}$ observed that the objective measurements of AA obtained using an open-field, infra-red optometer in 236 subjects were significantly lower than those found using the push-up method.
Bland-Altman analysis does not say whether agreement is sufficient or suitable to use a certain method but simply quantifies the range of agreement within which $95 \%$ of the differences between one and another method are included. It is the clinical goal that could define whether the agreement is wide or narrow for the purpose. The Bland-Altman plot only defines the intervals of agreement, and it does not mention whether those limits are acceptable or not. The acceptable limits should be defined prior, based on clinical necessity or other goals. If the line of the mean difference is not in the interval, there is a significant systemic difference.

\section{Limitations of the study}

There are several limitations to this study. Only normal and healthy optometry students were included in the study. These students were more accustomed to the instrumentation and may not have yielded typical clinical responses when compared to others. Symptomatic students did not participate in the study, and it is possible that the results from such a group would have been different.

Limitations of the dynamic retinoscopy techniques are that it takes longer to perform and the accuracy of the measurements will vary with skills of the examiner.

\section{Conclusion}

The push-up and push-down methods overestimate the true AA because of the relative magnification, while the minus lens method creates an abnormal viewing environment in which the target is stationary but the stimulus becomes increasingly minified. The AA measured using the two objective methods agreed with each other but differed from the subjectively measured AA. The objective measurements showed that AA is substantially less than that measured using the subjective methods. The results obtained using the modified and Pascal dynamic retinoscopy were well correlated and comparable suggesting that they could be used interchangeably in clinical settings. Subjective methods measure the nearest distance at which the patient can see clearly, while objective methods evaluate the actual increase in refractive power of the eye. So, subjective measurements of the AA may suggest that accommodation is present when it is not. This is because the eye's depth of field can cause subjective measurements to overestimate the true AA. Further research is needed to validate DR as the optimal routine clinical method to assess AA.

\section{Acknowledgements}

The authors thank the students who volunteered their time to participate in this study.

\section{Competing interests}

The authors declare that they have no financial or personal relationships that may have inappropriately influenced them in writing this article. 


\section{Authors' contributions}

All the authors made equal contributions to this study. S.D.M. designed the study, did the analysis and the first draft of the manuscript. K.L.L. performed the subjective measurements. M.D.N. performed the modified dynamic retinoscopy method. N.T.M. performed the Pascal heterodynamic retinoscopy.

\section{Funding information}

The work reported here was supported by the South African Medical Research Council to S.D.M. under Research Strengthening Capacity Building at Selected Universities.

\section{References}

1. Grosvenor T. Primary care optometry. 5th ed. London: Butterworth-Heinemann; 2007.

2. Rabbetts RB. Bennett and Rabbetts clinical visual optics. 3rd ed. London: Butterworth-Heinemann; 1998.

3. Tunnacliffe AH. Introduction to visual optics. 4th ed. London: ABDO; 2001.

4. Dubbelman $M$, van der Heijde GL, Weeber HA. Change in shape of the aging human crystalline lens with accommodation. Vis Res. 2005;45(1):117-132. https:// doi.org/10.1016/j.visres.2004.07.032

5. Eskridge JB, Amos JF, Bartlett JD. Clinical procedures in optometry. Philadelphia, PA: JP Lippincott Co.; 1991.

6. Benjamin WJ. Borish'S clinical refraction. Philadelphia, PA: W.B. Saunders Company; 1998. 7. Wolffsohn JS, Sheppard AL, Vakani S, Davies LN. Accommodative amplitude
required for sustained near work. Ophthalmic Physiol Opt. 2011;31:480-486. https://doi.org/10.1111/j.1475-1313.2011.00847.x

8. Young T. On the mechanism of the eye. Philos Trans R Soc Lond. 1801;91:23-88. https://doi.org/10.1098/rstl.1801.0004

9. Cogan D. Accommodation and autonomic nervous system. Arch Ophthalmol. 1937;18:739-766. https://doi.org/10.1001/archopht.1937.00850110055004

10. Plainis S, Charman WN, Pallikaris IG. The physiologic mechanism of accommodation. Cataract Refract Surg Today Europe. 2014 Apr 23-29.

11. Roy FH. Mechanism of accommodation in primates (letter). Ophthalmology 2001;108:1369-1371. https://doi.org/10.1016/S0161-6420(01)00664-9

12. Glasser A, Kaufman PL. The mechanism of accommodation in primates Ophthalmology 1999;106:863-872. https://doi.org/10.1016/S0161-6420(99) 00502-3

13. Levy NS. The mechanism of accommodation in primates. Ophthalmology 2000;107(4):627. https://doi.org/10.1016/S0161-6420(00)00039-7

14. Donders FC. On the anomalies of accommodation and refraction of the eye (transl. W.D. Moore). Boston, MA: Milford House Inc.; 1972.

15. Duane A. The accommodation and Donder's curve and the need to revisiting our ideas regarding them. JAMA. 1909;52:1992-1996. https://doi.org/10.1001/jama. 1909.25420510026002f

16. Duane A. Studies in monocular and binocular accommodation with their clinical applications. Trans Am Ophthalmol. 1922;20:132-157. https://doi.org/10.1016/ S0002-9394(22)90793-7

17. Duane A. An attempt to determine the normal range of accommodation at various ages, being a revision of Donder's experiments. Trans Am Ophthalmol Soc. 1908 11:634-641.

18. Duane A. Normal values of the amplitude of accommodation at all ages. JAMA. 1912;59:1010-1013. https://doi.org/10.1001/jama.1912.04270090254042

19. Jackson E. Amplitude of accommodation at different periods of life. Cal State J Med. 1907;5:163-166.

20. Kragha IKOK. Amplitude of accommodation: Population and methodological differences. Ophthalmic Physiol Opt. 1986;6(1):75-80. https://doi.org/10.1111/ j.1475-1313.1986.tb00703.x

21. Hamasaki D, Ong J, Marg E. The amplitude of accommodation in presbyopia. Am J Optom Arch Am Acad Optom. 1956;33:3-14. https://doi.org/10.1097/00006324195601000-00002

22. Anderson HA, Stuebing KK. Subjective vs objective accommodative amplitude: Preschool to presbyopia. Optom Vis Sci. 2014;91(11):1290-1301. https://doi. org/10.1097/OPX.0000000000000402

23. Hokoda SC, Ciuffreda KJ. Measurement of accommodative amplitude in amblyopia. Ophthalmic Physiol Opt. 1982;2(3):205-212. https://doi.org/10.1111/ j.1475-1313.1982.tb00178.x

24. Ostrin LA, Glasser A. Accommodation measurements in a prepresbyopic and presbyopic population. J Cataract Refract Surg. 2004;30(7):1435-1444. https:// doi.org/10.1016/j.jcrs.2003.12.045

25. Rutstein RP, Fuhr PD, Swiatocha J. Comparing the amplitude of accommodation determined objectively and subjectively. Optom Vis Sci. 1993;70(6):496-500. https://doi.org/10.1097/00006324-199306000-00008
26. Wold JE, Hu A, Chen S, Glasser A. Subjective and objective measurement of human accommodative amplitude. J Cataract Refract Surg. 2003;29(10):1878-1888. https://doi.org/10.1016/S0886-3350(03)00667-9

27. Otake $\mathrm{Y}$, Miyao $\mathrm{M}$, Ishihara $\mathrm{S}$, et al. An experimental study on the objective measurement of accommodative amplitude under binocular and natural viewing conditions. Tohoku J Exp Med. 1993;170(2):93-102. https://doi.org/10.1620/tjem. 170.93

28. Anderson HA, Hertz G, Glasser A, Stuebing KK, Manny RE. Minus-lens-stimulated accommodative amplitude decreases sigmoidally with age: A study of objectively measured accommodative amplitudes from age 3. Invest Ophthalmol Vis Sci. 2008;49(7):2919-2926. https://doi.org/10.1167/iovs.07-1492

29. Wagstaff DF. The objective measurement of the amplitude of accommodation Part vi and vii. Optician. 1966;151:323-326,431-436.

30. Allen PE, Evans BJ. Clinical measurement of amplitude of accommodation: A review. Optom Pract. 2014:15(3):75-85.

31. Atchison DA, Capper EJ, McCabe KL. Critical subjective measurement of amplitude of accommodation. Optom Vis Sci. 1994;71(11):699-706. https://doi.org/ 10.1097/00006324-199411000-00005

32. Rosenfield M, Cohen AS. Push-up amplitude of accommodation and target size (letter). Ophthalmic Physiol Opt. 1995;15(3):231-232. https://doi.org/10.1016/ 0275-5408(95)90576-N

33. Atchison DA, Charman WN, Woods RL. Subjective depth-of-focus of the eye. Optom Vis Sci. 1997; 74(7):511-520. https://doi.org/10.1097/00006324-199707000-00019

34. Momeni-Moghaddam H, Wolffsohn J, Azimi A, Babaei-Malekkolaei E. Effect of target distance on accommodative amplitude measured using the minus-lens technique. Clin Exp Optom. 2013;97(1):62-65. https://doi.org/10.1111/cxo.12090

35. Koslowe K, Glassman T, Tzanani-Levi C, Shneor E. Accommodative amplitude determination: Pull-away versus push-up methods. Optom Vis Dev. 2010;41(1): 28-32.

36. Woehrle MB, Peters RJ, Frantz KA. Accommodative amplitude determination: Can we substitute the pull-away for the push-up method? J Optom Vis Dev. 1997; 28(4):246-250.

37. Pollock J. Accommodation measurement: Clear or blurred? Aust Orthopt J. 1989; 25:20-22.

38. Chen AH, O'Leary DJ. Validity and repeatability of the modified push-up method for measuring the amplitude of accommodation. Clin Exp Optom. 1998;81(2): 63-71. https://doi.org/10.1111/j.1444-0938.1998.tb06628.x

39. Rosenfield M, Cohen AS. Repeatability of clinical measurement of the amplitude of accommodation. Ophthalmic Physiol Opt. 1996;16(3):247-249. https://doi.org/ of accommodation. Ophthalmic
$10.1016 / 0275-5408(95) 00093-3$

40. Antona B, Barra F, Barrio A, Gonzalez E, Sanchez I. Repeatability, interexaminer and agreement in amplitude of accommodation measurements. Graefes Arch Clin Exp Ophthalmol. 2009;247(1):121-127. https://doi.org/10.1007/s00417-008-0938-9

41. Taub MB, Shallo-Hoffmann J. A comparison of three clinical tests of accommodation amplitude to Hoffstetter's norm to guide diagnosis and treatment. Optom Vis Dev. 2012;43(4):180-190.

42. Sharma IP. RAF near point rule for near point of convergence - A short review. Ann Eye Sci. 2017;2(16). https://doi.org/10.21037/aes.2017.02.05

43. Majumder C. Comparison of amplitude of accommodation in different vertical viewing angles. Optom Vis Perf. 2015;3(5):276-280.

44. Leon AA, Medrano SM, Rosenfield M. A comparison of the reliability of dynamic retinoscopy and subjective measurements of amplitude of accommodation. Ophthalmic Physiol Opt. 2012;32(2):133-141. https://doi.org/10.1111/j.14751313.2012.00891.x

45. Leon AA, Estrada JM, Rosenfield. Age and the amplitude of accommodation measured using dynamic retinoscopy. Ophthalmic Physiol Opt. 2016;36(1):5-12. https://doi.org/10.1111/opo.12244

46. Scheiman M, Wick B. Clinical management of binocular vision: Heterophoria, accommodative, and eye movement disorders. 3rd ed. Philadelphia, PA: Lippincott Williams \& Wilkins; 2008.

47. Rosenfield M, Portello JK, Blustein GH, Jang C. Comparison of clinical techniques to assess the near accommodative response. Optom Vis Sci. 1996;73(6):382-388. to assess the near accommodative response. Optom V
https://doi.org/10.1097/00006324-199606000-00005

48. Whitefoot $\mathrm{H}$, Charman WN. Dynamic retinoscopy and accommodation. Ophthal Physiol Opt. 1992;12(1):8-17. https://doi.org/10.1111/j.1475-1313.1992.tb00247.x

49. Locke LC, Somers W. A comparison study of dynamic retinoscopy techniques. Optom Vis Sci. 1989;66(8):540-544. https://doi.org/10.1097/00006324198908000-00009

50. Pascal JI. Neutralization in dynamic retinoscopy. Br J Ophthalmol. 1931;15(10):589590. https://doi.org/10.1136/bjo.15.10.589

51. Ghasemi A, Zahediasl S. Normality tests for statistical analysis: A guide for nonstatisticians. Int J Endocrinol Metab. 2012;10(2):486-489. https://doi.org/10.5812/ ijem.3505

52. Razali NM, Wah YB. Power comparisons of Shapiro-Wilk, Kolmogorov-Smirnov, Lilliefors and Anderson-Darling tests. J Stat Model Anal. 2011;21(1):21-33.

53. Carkeet A. Exact parametric confidence intervals for Bland-Altman limits of agreement. Optom Vis Sci. 2015;92(3):e71-e80. https://doi.org/10.1097/OPX. 0000000000000513

54. Carkeet A, Goh YT. Confidence and coverage for Bland-Altman limits of agreement and their approximate confidence intervals. Stat Methods Med Res. 2016;25:1-16.

55. Lopez-Gil N, Fernandez-Sanchez V, Thibos LN, Montes-Mico R. Objective amplitude of accommodation computed from optical quality metrics applied to wavefront outcomes. J Optom. 2009;2(4):223-234. https://doi.org/10.3921/ joptom.2009.223 
56. Seidemann A, Schaeffel F. An evaluation of the lag of accommodation using photorefraction. Vision. 2003;43(4):419-430. https://doi.org/10.1016/S0042-6989 (02)00571-0

57. Goss DA. Clinical accommodation testing. Curr Opin Ophthalmol. 1992;3(1):78-82. https://doi.org/10.1097/00055735-199202000-00011

58. Rosenfield M, Gilmartin B. Effect of target proximity on the open-loop accommodative response. Optom Vis Sci. 1990;67(2):74-79. https://doi.org/ 10.1097/00006324-199002000-00002
59. Ang RET, Sarmiento JAS, Remo JTM, Martinez GHA, Canilao LMB. Measurement of accommodative amplitude using wavefront aberrometer. Philipp J Ophthalmol. 2015;40(1):3-10.

60. Win-Hall DM, Glasser A. Objective accommodation measurements in prepresbyopic eyes using an autorefractor and an aberrometer. J Cataract Refract Surg. 2008; 34(5):774-784. https://doi.org/10.1016/j.jcrs.2007.12.033

61. Woodhouse JM, Meades JS, Leat SJ, Saunders KJ. Reduced accommodation in children with Down syndrome. Invest Ophthalmol Vis Sci. 1993;34:2382-2387. 